\title{
Using the steatocrit to determine optimal fat content in modular feeds
}

\author{
M O Rawashdeh, D R Lloyd, J W L Puntis, G A Brown,, I W Booth
}

\begin{abstract}
The steatocrit was measured in infants with protracted diarrhoea who were receiving intragastric modular feeds. Measurements were made when fat intake was constant to determine steatocrit variability and during increases in fat intake to determine fat tolerance limits. Steatocrit variability was expressed as the range between a subject's lowest and highest steatocrit value. The median betweenstool and between-day variabilities were $2 \%$ and $11 \%$ respectively.

Variability was also measured in seven healthy breast fed infants. In the five still displaying appreciable physiological steatorrhoea, the between-day variability (median $8 \%$ ) and between-stool variability (median $9 \%$ ) were not significantly different from the protracted diarrhoea group.

In the fat tolerance investigations in the protracted diarrhoea group, the steatocrit increased with increases in the module fat content. Fat intake and steatocrit were significantly positively related. A significant negative correlation was seen between steatocrit and weight gain, the latter becoming negligible at steatocrit values around $30 \%$.
\end{abstract}

Modular feeds have an important role in the management of infants with severe, protracted diarrhoea. Each macronutrient can be varied independently, both quantitatively and qualitatively, so that modular feeding often obviates the need for parenteral nutrition. ${ }^{1}$ For example, in infants unable to tolerate the glucose polymer concentration present in protein hydrolysate based feeds, an isocaloric quantity of carbohydrate can be provided in a modular feed as a mixture of glucose polymer and sucrose without exceeding the carbohydrate tolerance limit.

Tolerance limits for carbohydrate can be established readily by monitoring stools for the first appearance of excess sugar using the Clinitest tablet test (Ames Laboratories) of Kerry and Anderson. ${ }^{2}$ Fat is a more energy dense and therefore more effective energy source and there is evidence to suggest that it is better tolerated than carbohydrate during protracted diarrhoea. ${ }^{3}$ However, fat intolerance, unlike carbohydrate intolerance, is not necessarily marked by a dramatic increase in stool volume or other clinical signs and steatorrhoea may have important adverse effects. ${ }^{4}$ There is therefore a need for a rapid and simple test for stool fat content, analogous to the Clinitest for carbohydrate, for use in patients receiving modular feeds.
Phuapradit et al, measuring fat excretion in the newborn, showed a significant positive correlation between the stool fat concentration determined chemically and by a simple, rapid microvolumetric measurement which they named the steatocrit. ${ }^{5}$ In the steatocrit, the stool fat content is determined by measuring the thickness of the fat layer produced by centrifugation of a stool and water homogenate and expressing it as a percentage of the total nonaqueous phase (fat plus stool solids layers). The effect of the variable stool water content is therefore eliminated.

The procedure was validated by showing a significant positive correlation between the steatocrit value and the stool fat content determined chemically. ${ }^{5}$ Other workers showed a positive correlation between the steatocrit value and the fat content of a three day stool collection but used the same stool homogenate for both analyses. ${ }^{6}$ The correlation between the fat content of a stool collection (72 hours or longer) and a single random stool steatocrit measured during the collection period has not been determined and there is very little information available on between-stool variation of the steatocrit and none on between-day variation. The clinical value of the single random stool steatocrit therefore remains unknown.

In this study the steatocrit has been evaluated for a specific role which did not require previous validation of the relation between the fat content of a sample from a single stool and the fat content of several days' stool collection. We have used it to determine the fat intake optimum for infants with protracted diarrhoea who were receiving modular feeds by continuous intragastric infusion. We have also determined the between-stool and between-day variability of the steatocrit and compared the results with steatocrit variability in a group of normal neonates.

\section{Subjects and methods}

INFANTS RECEIVING MODULAR FEEDS

Steatocrit values were determined in a group of 14 infants with protracted diarrhoea aged 1-9 months (median 4 months). Nine had postgastroenteritis syndrome, four were recovering from necrotising enterocolitis, and one had cystic fibrosis. All were in hospital receiving modular feeds by continuous nasogastric infusion. Body weights were measured daily.

NORMAL INFANTS

Steatocrit values were determined in a group of 
seven healthy infants, aged 12-63 days, who were at home and exclusively breast fed. Babies of this age were selected because they had steatorrhoea without the potential complications of gastrointestinal pathology.

\section{MODULAR FEEDS}

The 14 infants with protracted diarrhoea were fed by continuous intragastric infusion of a modular feed in which protein was provided as comminuted chicken (Cow and Gate) $30 \mathrm{~g} / 100$ $\mathrm{ml}$, carbohydrate as a variable mixture of sucrose and glucose polymer (Maxijul, Scientific Hospital Supplies), and fat as an arachis oil emulsion(Calogen, Scientific Hospital Supplies). The module carbohydrate content $(10 \mathrm{~g} / 100 \mathrm{ml})$ was constant throughout for all subjects and none had evidence of carbohydrate malabsorption at any time.

STOOL COLLECTION REGIMEN

Between-stool variability was determined in 11 of the 14 infants with protracted diarrhoea by sampling all stools passed over a single 24 hour period. Between-day variability was determined in nine of the group by collecting stool samples once daily for at least three consecutive days. The variability studies were conducted when fat administration was at a constant daily value. The fat intake-steatocrit relationship was determined during periods of daily incrementation of the module fat content.

In the healthy infants, all stools were sampled over 72 hours. Between-stool variability was assessed using stools from the day which produced the most stool specimens. Betweenday variability was assessed using the first stool collected on each of the three days.

\section{STEATOCRIT PROCEDURE}

The method of Phuapradit $e t$ al was used with the following modifications. ${ }^{5}$ The stool homogenate concentration was reduced to a stool: water ratio of $1: 3 \mathrm{w} / \mathrm{w}$ and homogenates were prepared using a Potter-Elvehjem glass and Teflon homogeniser $(0.15 \mathrm{~mm}$ clearance $)$ instead of the pestle and mortar with sand procedure originally described. A lipid stain (rhodamine 6G) was added to the stool homogenate for improved demarcation of the fat-aqueous and solid-aqueous phase boundaries after centrifugation. Fat and solid phase thickness was measured with a dial caliper calibrated to $0 \cdot 1$ $\mathrm{mm}$.

In some stool samples the quantity and nature of the lipid present resulted in the separation of a 'waxy' lipid material, during homogenisation, which adhered to the homogeniser surfaces leading potentially to an under estimation of fat content. Heating the stool-water mixture briefly to $70^{\circ} \mathrm{C}$ in a water bath before homogenisation dispersed the adherent lipid and the heating step was used therefore for all stool samples in the study. In a validation experiment in which steatocrit values were determined in five waxy stools and 36 non-waxy stools, with and without the heating step, steatocrit values were signifi- cantly increased $(\mathrm{p}<0.05)$ in the waxy stools after heating but not in the non-waxy samples.

\section{STEATOCRIT METHOD IMPRECISION}

Imprecision was determined by separate batch, duplicate analysis of 16 stools. The stools were included in four batches analysed over a period of seven days. The 16 duplicate mean steatocrit values covered the range $7-58 \%$. The coefficient of variation was $17 \cdot 2 \%$. The range of differences between the 16 duplicate analyses was $0-23 \%$, median difference $3 \%$.

The coefficient of variation produced by homogenising 16 samples taken from the same stool was $13 \cdot 1 \%$. The median steatocrit value for the 16 replicate analyses was $16 \%$, range $13-20 \%$.

\section{STEATOCRIT VARIABILITY}

Steatocrit variability was expressed as the difference between the highest and lowest steatocrit value produced by a baby in the interval of interest. If, for example, the lowest and highest values were $2 \%$ and $19 \%$ respectively, then variability was recorded as $17 \%$.

\section{STATISTICAL EVALUATION}

All statistical evaluations were non-parametric. The degree of association between two measurements and significance was determined using the Spearman rank correlation coefficient. The Mann-Whitney U test and Wilcoxon matched pairs signed rank test were used to determine the significance of differences between unpaired and paired data groups respectively.

\section{Results}

INFANTS WITH PROTRACTED DIARRHOEA

The between-stool steatocrit variation is shown in fig $1 \mathrm{~A}$. In 11 infants on a constant fat intake, from whom all stools were sampled within a 24 hour period, the highest variability value was $14 \%(44-58 \%)$ and the median value for the group was $2 \%$.

The between-day variation is shown in fig 2 . In nine infants in whom stool samples were collected once daily for 3-5 days while on a constant fat intake, the highest variability value was $21 \%$ and the median value was $11 \%$.

The relation between steatocrit value and fat intake in the fat tolerance investigations is shown in fig 3. In the 11 infants investigated during periods of daily increases in modular fat content, the steatocrit values increased within 24 hours after each fat increment except in three infants where there was a threshold phenomenon, the steatocrit values increasing only after several increments in fat intake. The positive correlation between fat intake and steatocrit values, using the group pooled data, was statistically significant $(r=0.54, p<0.01)$.

Using data derived from all 14 babies, there was a significant negative correlation $(r=-0 \cdot 71$, $\mathrm{p}<0.01$ ) between the mean daily body weight increment $(\mathrm{g} / \mathrm{kg}$ body weight $/ 100 \mathrm{kcal} /$ day) and a mean steatocrit value derived from seven daily 

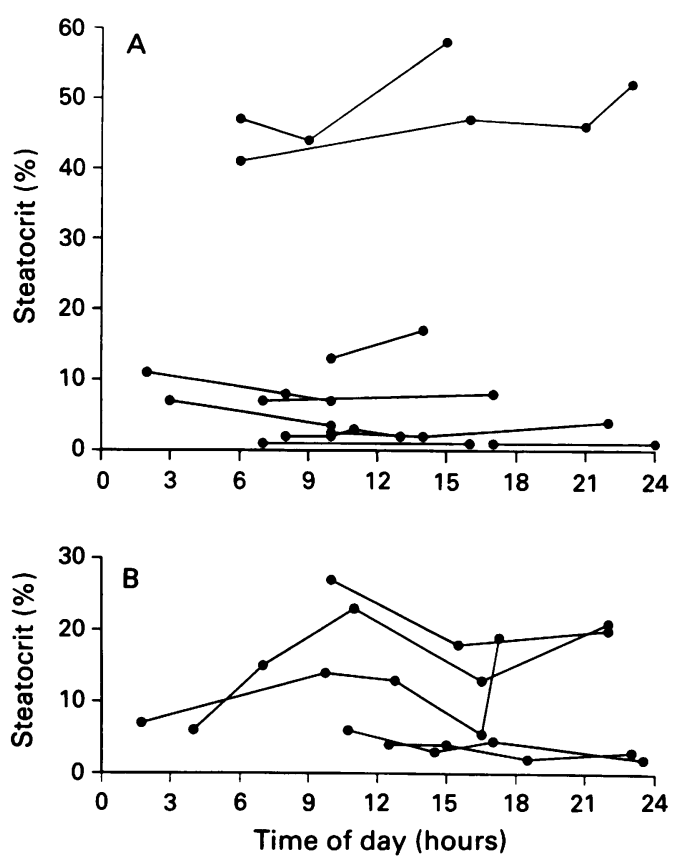

Figure 1 Between-stool variability of the steatocrit in $(A)$ 11 infants with protracted diarrhoea and $(B)$ in five healthy, breast fed neonates. Samples were collected from consecutive stools passed on the same day. Each line joins observations from a single subject.

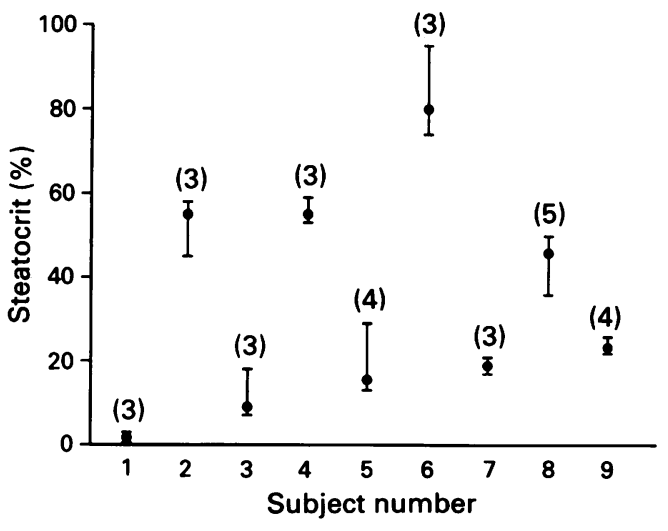

Figure 2 Between-day variability of the steatocrit in nine infants with protracted diarrhoea. Stools were sampled once daily for 3-5 days (number in parenthesis). The vertical plots show the range and median value for the individual infant.

observations, while on a constant fat intake (fig 4). The baby with the highest mean steatocrit value actually lost weight during the study period.

\section{NORMAL INFANTS}

The number of stools collected over the 72 hour collection period in each baby varied between 4 and 14 (median 12), the larger numbers being collected from the younger babies. The overall range of steatocrit values in the 71 stool samples was $0-26 \%$ and the highest single day, betweenstool variability of $17 \%(6-23 \%)$ was seen in the youngest baby (fig 1B). The higher steatocrit values were seen in the younger babies and there was a significant negative correlation (fig 5) between the individual baby's 72 hour median steatocrit value and age $(r=-0.893$, $\mathrm{p}=0.01$ ).

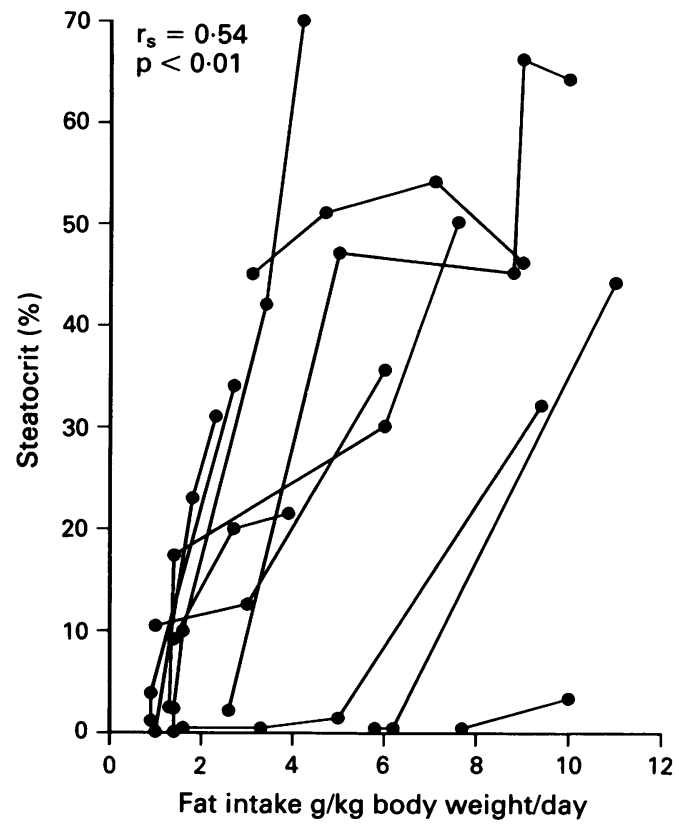

Figure 3 Steatocrit values determined in 11 infants with protracted diarrhoea during progressive daily increases of the module feed fat content. Each line joins observations from a single subject. Spearman rank correlation of the pooled data produced a coefficient of $0.54, p<0.01$.

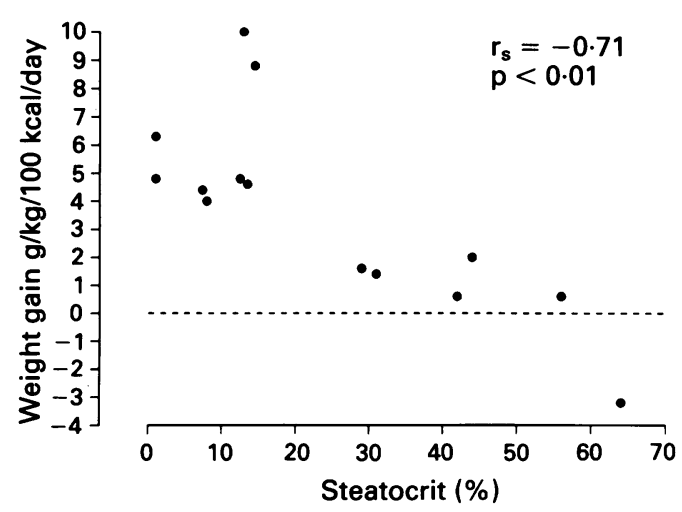

Figure 4 The steatocrit and weight gain relation in 14 infants with protracted diarrhoea. Spearman rank correlation produced a coefficient of $0 \cdot 71, p<0 \cdot 01(100 \mathrm{kcal}=418 \mathrm{~kJ})$.

Steatocrit values were $2 \%$ or less in the oldest two babies aged 6 and 9 weeks. Between-stool variability in the other five babies, who were less than one month of age, was $2-17 \%$ with a median of $9 \%$. Their between-day variability range was $2-15 \%$, median $18 \%$.

The between-stool and between-day steatocrit variabilities were not significantly different either within the two groups or between the two groups.

\section{Discussion}

Some measure of faecal fat excretion is essential for the objective assessment of fat digestion and absorption. Where the assessment is required at frequent intervals, the chemical methods for determining stool fat content are too cumbersome and a simple, rapid alternative is required. The steatocrit fulfils this need and is technically 


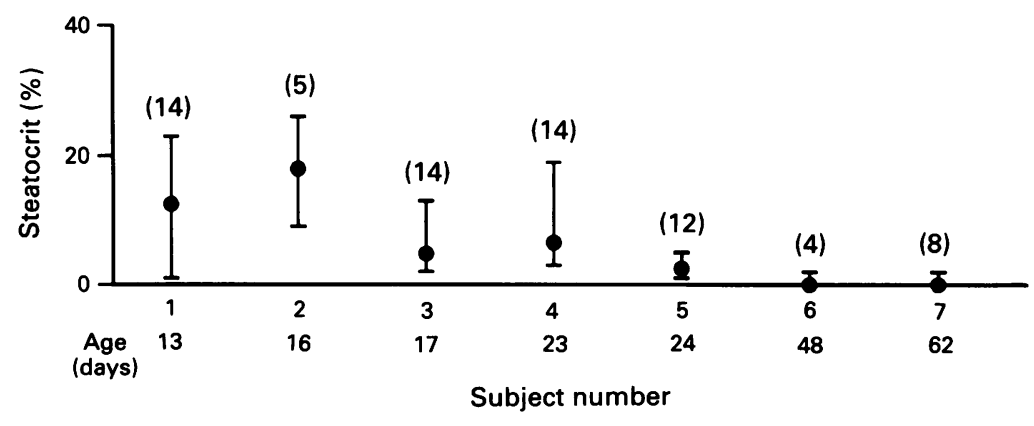

Figure 5 Between-stool variability of the steatocrit in 7 healthy, breast fed infants shown in age order. Samples were collected from stools passed over a 72 hour period. The vertical lines show the range and median value for each infant. The median values showed a significant negative correlation with age. Numbers of parentheses indicate number of stools passed during the 72 hour collection.
The variability found in most of the infants was sufficient to suggest that a single random stool steatocrit determination would not be sufficient for a precise index of steatorrhoea. The mean of several separate stool measurements would be necessary. The between-stool and between-day variability were not significantly different in either group of babies and an index of fat excretion could therefore be produced by sampling stools over any convenient interval which produced sufficient stools.

The rapidity of response to the increases in dietary fat intake, which was consistent with data on mouth to anus transit times in infants, ${ }^{11}$ shows the importance of careful control and constancy of fat intake if the steatocrit is used for screening for steatorrhoea. It is also important to ensure that an adequate fat intake is maintained during the stool sampling period.

In our particular clinical context, single steatocrit values proved to be sufficient. The rapid response and close relation to increases in dietary fat provided the information necessary to determine fat tolerance, that is, the point at which further increases in modular fat content provoked a sharp increase in steatocrit value.

In three infants, the fat intake and coincident steatocrit were less closely related than in the rest of the group due to a threshold phase in their fat intake-steatocrit correlation. The steatocrit did not rise sharply after the initial increases in module fat content and their steatocrit values were lower than the rest of the group for a given fat intake. This threshold pattern of response to increasing fat intake has been demonstrated in healthy adults. ${ }^{12}$ The inflection in the correlation presumably indicates the fat tolerance limit, that is, the point at which the fat load saturates the digestionabsorption mechanisms. However, even above this inflection point, fat absorption was more efficient than in the remainder of the group, the increase in steatocrit being smaller for a given increase in fat intake. The fat intake:steatocrit regression factor therefore may be an index of the severity of malabsorption or its underlying pathology, the higher the factor the more severe the malabsorption.

A further function of the steatocrit that emerged from the study was its predictive value with respect to weight gain, high values being associated with low weight gains irrespective of the underlying pathology. The slope of this relationship suggested that further increments of fat intake were of limited nutritional value when the steatocrit was $30 \%$ or greater. In the context of this study, this correlation also obviates the need to validate the steatocrit by showing a positive correlation with a reference chemical procedure for stool fat measurement, on a 72 hour stool collection for example. If steatocrit measurements have already shown a statistically significant correlation with weight gain (an objective measurement indicative of patient welfare) there is no longer a need to relate it to other fat measurement methods, the relevance to weight gain being a more fundamental relationship in the management of children with protracted diarrhoea.

The steatocrit literature contains very little in 15 nor ${ }^{8}$. however was determined for each baby using data from as few as two stools with consequent loss of validity. 
satisfactory validation or error data for comparison with our modified procedure. Phuapradit $e t$ al determined method imprecision by repeated analysis of the same two stool homogenates, of high and low steatocrit value, producing coefficients of variation of $2 \%$ and $12 \%$ respectively. ${ }^{5}$ The error associated with homogenate production and any non-homogeneity of stool sample was therefore not included in the assessment.

In a further validation experiment, coefficients of variation of $4 \cdot 5-15 \cdot 7 \%$, for steatocrit levels of $5-35 \%$, were found in normal stool samples to which varying quantities of butter had been added to stimulate steatorrhoea. ${ }^{8}$ Our coefficient of variation, determined from between-batch duplicate differences in stool samples with a wide range of steatocrit values $(7-58 \%)$, was a little higher $(17 \cdot 2 \%)$ but incorporated the error of the complete procedure over a greater range of steatocrit values.

The relation of steatocrit value and the nature of the stool lipid is so far uninvestigated. The type of fatty acid present in stools during steatorrhoea reflects the dietary fatty acid and will be variable, particularly if therapeutic diets are taken into account. Unsaturated and medium chain fatty acids are liquid at ambient temperatures. Where diets contain large quantities of either lipid any non-absorbed fatty acids in stool should separate more efficiently, when centrifuged for steatocrit measurement, than saturated fatty acids, the predominant dietary fatty acids, which are solid at room temperature. Medium chain fatty acids however would be unlikley to appear in stools in appreciable quantities, the rationale for their use being ready absorption in circumstances where the digestion and absorption of long chain triglyceride has failed.
The statistically significant correlations that have been demonstrated between steatocrit value and stool fat content, measured by gravimetric $^{5}$ or chemical analysis, ${ }^{6}$ in large subject groups, suggest that any effect of variation in dietary lipid quality is insufficient to obscure the correlation.

With thanks to Gregory Gale and Fraser Brown who participated without complaint in the normal infant group.

1 Larcher VF, Shepherd R, Francis DEM, Harries JT. Protracted diarrhoea in infancy. Arch Dis Child 1977:52: 597-605.

2 Kerry KR, Anderson CM. A ward test for sugar in faeces. Lancet 1964;ii:981-2.

3 Galeano NF, Lepage G, Leroy C, Belli D, Levy E, Roy CC. Comparison of two special infant formulas designed for the treatment of protracted diarrhoea. 7 Pediatr Gastroenterol treatment of protracte

4 Bright-Asare $\mathrm{P}$, Binder $\mathrm{HJ}$. Stimulation of colonic secretion of water and electrolytes by hydroxy fatty acids. Gastroof water and electrolytes
enterology 1973;64:81-8.

5 Phuapradit P, Narang A, Mendonca P, Harris DA, Baum JD. The steatocrit: a simple method for estimating stool fat content in newborn infants. Arch Dis Child 1981;56:725-7.

6 Colombo R, Maiavacca R, Ronchi M, Consalvo E, Amorett A, Giunta A. The steatocrit: a simple method for monitoring fat malabsorption in patients with cystic fibrosis. $\mathcal{F}$ Pediatr Gastroenterol Nutr 1987;6:926-30

7 Fomon SJ, Ziegler EE, Thomas LN, Jensen RL, Filer LJ. Excretion of fat by normal full-term infants fed various milks and formulas. Am f Clin Nutr 1970;23:1299-313.

8 Iacono G, Carroccio A, Cavataio F, et al. Steatocrit test: normal range and physiological variations in infants. $\mathcal{F}$ Pediatr Gastroenterol Nutr 1990;11:53-7.

9 Walters MP, Kelleher J, Gilbert J, Littlewood JM. Clinical monitoring of steatorrhoea in cystic fibrosis. Arch Dis Child monitoring of steatc

10 Picciano MF, Guthrie HA, Sheehe DM. The cholesterol content of human milk. Clin Pediatr (Phila) 1978;17: content

11 Rubaltelli FF, Largajolli G. Effect of light on gut transit time in jaundiced newborns. Acta Paediatr Scand 1973;62: 146-8.

12 Saunders DR, Sillery JK. Absorption of triglyceride by human small intestine: dose response relationships. $A m \mathcal{F}$ Clin Nutr 1988;48:988-91. 